\title{
Antimicrobial susceptibilities of aerobic and facultative gram-negative bacilli isolated from Chinese patients with urinary tract infections between 2010 and 2014
}

Qiwen Yang ${ }^{1+}$, Hui Zhang ${ }^{1+}$, Yao Wang ${ }^{1}$, Zhipeng $X u^{1}$, Ge Zhang ${ }^{1}$, Xinxin Chen ${ }^{1}$, Yingchun $X u^{1 *}$, Bin $\mathrm{CaO}^{2}$, Haishen Kong ${ }^{3}$, Yuxing $\mathrm{Ni}^{4}$, Yunsong $\mathrm{Yu}^{5}$, Ziyong Sun ${ }^{6}$, Bijie Hu${ }^{7}$, Wenxiang Huang ${ }^{8}$, Yong Wang ${ }^{9}$, Anhua $\mathrm{Wu}^{10}$, Xianju Feng ${ }^{11}$, Kang Liao ${ }^{12}$, Yanping Luo ${ }^{13}$, Zhidong Hu${ }^{14}$, Yunzhuo Chu ${ }^{15}$, Juan Lu ${ }^{16}$, Jianrong Su${ }^{17}$, Bingdong Guil ${ }^{18}$, Qiong Duan ${ }^{19}$, Shufang Zhang ${ }^{20}$, Haifeng Shao ${ }^{21}$ and Robert E. Badal ${ }^{22}$

\begin{abstract}
Background: The objective of this study was to investigate the distribution and susceptibility of aerobic and facultative Gram-negative bacilli isolated from Chinese patients with UTIs collected within $48 \mathrm{~h}$ (community acquired, CA) or after $48 \mathrm{~h}$ (hospital acquired, HA) of hospital admission.
\end{abstract}

Methods: From 2010 to 2014, the minimum inhibitory concentrations (MICs) of 12 antibiotics for 4,332 aerobic and facultative Gram-negative bacilli, sampled in 21 hospitals in 16 cities, were determined by the broth microdilution method.

Results: Enterobacteriaceae composed $88.5 \%$ of the total isolates, with Escherichia coli (E. coli) (63.2\%) the most commonly isolated species, followed by Klebsiella pneumoniae (K. pneumoniae) (12.2\%). Non-Enterobacteriaceae accounted for only $11.5 \%$ of all isolates and included mainly Pseudomonas aeruginosa (P. aeruginosa) (6.9\%) and Acinetobacter baumannii (A. baumannii) (3.3\%). Among the antimicrobial agents tested, the susceptibility rates of $E$. coli to the two carbapenems, ertapenem and imipenem as well as amikacin and piperacillin-tazobactam ranged from 92.5 to $98.7 \%$. Against K. pneumonia, the most potent antibiotics were imipenem (92.6\% susceptibility), amikacin (89.2\% susceptibility) and ertapenem (87.9\% susceptibility).

Although non-Enterobacteriaceae did not show high susceptibilities to the 12 common antibiotics, amikacin exhibited the highest in vitro activity against $P$. aeruginosa over the 5-year study period, followed by piperacillintazobactam, imipenem, ceftazidime, cefepime, ciprofloxacin, and levofloxacin. The Extended Spectrum BetaLactamase (ESBL) rates decreased slowly during the 5 years in E. coli from $68.6 \%$ in 2010 to $59.1 \%$ in 2014, in K. pneumoniae from 59.7 to 49.2\%, and in Proteus mirabilis (P. mirabilis) from 40.0 to 26.1\%. However, the ESBL rates were different in 5 regions of China (Northeast, North, East, South and Middle-China).

(Continued on next page)

\footnotetext{
* Correspondence: xuyingchunbm@163.com

${ }^{\dagger}$ Equal contributors

'Department of Clinical Laboratory, Peking Union Medical College Hospital,

Peking Union Medical College, Chinese Academy of Medical Sciences, Beijing 100730, China

Full list of author information is available at the end of the article
} 
(Continued from previous page)

Conclusion: E. coli and K. pneumonia were the major pathogens causing UTIs and carbapenems and amikacin retained the highest susceptibility rates over the 5-year study period, indicating that they are good drug choices for empirical therapies, particularly of CA UTIs in China.

Keywords: Urinary tract infections, Extended spectrum beta-lactamases (ESBLs), Carbapenems, Antimicrobial resistance

\section{Background}

Several national and international surveillance programs have been initiated for monitoring susceptibilities of clinically important pathogens in urinary tract infections (UTIs) [1-3]. The Study for Monitoring Antimicrobial Resistance Trends (SMART) is a surveillance program designed to monitor globally susceptibilities of aerobic and facultative Gram-negative bacilli collected from intra-abdominal infections and UTIs (initiated in 2002) [4]. UTIs are frequently encountered in clinical practice and include uncomplicated and complicated pyelonephritis, ureteritis, cystitis and urethritis [5]. The etiologies of these infections arise from Gram-negative bacilli, especially Enterobacteriaceae, and some Gram-positive bacteria [6]. During the last decade, multidrug-resistant Gram-negative Enterobacteriaceae have become a challenge for physicians [7] and particularly E. coli and $K$. pneumonia strains isolated from UTIs have been reported to increasingly produce ESBLs in the recent years [8-10]. The choice of an empiric UTI antimicrobial therapy should be based on knowledge of the pathogen distribution and the resistance extent of common microorganisms, in addition to hospital-specific resistance patterns particularly for HA infections. This study, as part of the global SMART project, focused on ESBLproducing rates of UTI isolates from 21 centers in 16 Chinese cities between 2010 and 2014 and on UTI derived sample resistance rates against carbapenems, a combination of drugs containing penicillins with $\beta$ lactamase inhibitors, a cephamycin, an aminoglycoside, 3rd and $4^{\text {th }}$ generation cephalosporins as well as 2nd generation fluoroquinolones, in order to provide guidance for antimicrobial therapies of IAIs.

\section{Methods}

\section{Clinical isolates}

During our study period (2010-2014), a total of 4,332 aerobic and facultative Gram-negative bacilli were consecutively isolated from patients with UTIs in 21 hospitals sited in 16 Chinese cities (Beijing, Shanghai, Hangzhou, Nanjing, Shenyang, Tianjin, Wuhan, Changsha, Jinan, Zhengzhou, Guangzhou, Nanchang, Haikou, Harbin, Changchun and Chongqing).

All isolates were cultured from specimens collected from patients who met both clinical and laboratory criteria of urinary tract infections $(3,994$ from clean catch midstream urine, 154 from urinary bladder, 136 from ureter, 29 from kidney, 13 from urethra, 6 from prostate). Duplicate isolates (same species and genus from one patient) were excluded.

Standard methods were used by the participating clinical microbiology laboratories for initial bacteria identification, and re-identification was carried out by a central laboratory (Peking Union Medical College Hospital) using Vitek 2 Compact (2010-2011) (Biomerieux, France) and MALDI-TOF MS (2012-2014) (Vitek MS, Biomerieux, France).

Isolates were considered to be community-associated (CA) if they were recovered from a specimen taken less than $48 \mathrm{~h}$ after the patient was admitted to a hospital, and hospital-associated (HA) if the specimen was taken 48 or more hours after hospital admission, as previously described [11].

\section{Antimicrobial susceptibility test method}

Minimum inhibitory concentration (MIC) determinations were performed in a central lab using dehydrated MicroScan broth microdilution panels (Siemens Medical Solutions Diagnostics (West Sacramento, CA) according to Clinical and Laboratory Standards Institute (CLSI) guidelines [12] and susceptibility interpretations were based on clinical CLSI breakpoints [13]. Twelve commonly used antimicrobial agents for UTI treatments were analyzed namely, imipenem (IPM), ertapenem (EPM), ceftriaxone $(\mathrm{CRO})$, cefotaxime (CTX), ceftazidime (CAZ), cefoxitin (FOX), cefepime (FEP), piperacillin-tazobactam (TZP), ampicillin-sulbactam (SAM), amikacin (AMK), ciprofloxacin (CIP) and levofloxacin (LVX). For each batch of MIC testing, the reference strains E. coli ATCC 25922, P. aeruginosa ATCC 27853 and K. pneumonia ATCC 700603 were used as quality controls. Results were only included in the analysis when corresponding quality control isolate test results were in accordance with CLSI guidelines and therefore within an acceptable range.

\section{Extended-spectrum $\beta$-lactamases (ESBLs) detection}

Phenotypic identification of ESBL production in E.coli, $K$. pneumonia, Klebsiella oxytoca (K. oxytoca), and P. mirabilis was carried out according to CLSI recommended methods [13]. If cefotaxime or ceftazidime MICs were $\geq 2 \mu \mathrm{g} / \mathrm{mL}$, the MICs of cefotaxime + clavulanic acid $(4 \mu \mathrm{g} / \mathrm{mL})$ or 
ceftazidime + clavulanic acid $(4 \mu \mathrm{g} / \mathrm{mL})$ were comparatively determined. ESBL production was defined as a $\geq 8$-fold decrease in MICs for cefotaxime or ceftazidime tested in combination with clavulanic acid, compared to their MICs without clavulanic acid.

\section{Statistical analysis}

The susceptibility of all gram-negative isolates combined was calculated using breakpoints appropriate for each species and assuming 0\% susceptible for species with no breakpoints for any given drug. Ninety-five percent confidence intervals were calculated using the adjusted Wald method; linear trends of ESBL rates in different years were assessed for statistical significance using the CochranArmitage test and comparison of ESBL rates in 6 different geographic areas were assessed using Chi-square test. $P$ values $<0.05$ were considered statistically significant.

\section{Results}

\section{Distribution of organisms from urinary tract infection}

A total of 4,332 isolates were collected from UTIs between 2010 and 2014. The highest distribution of bacteria was $E$. coli, which accounted for $63.2 \%$ (2,737 strains), followed by $K$. pneumonia (12.2\%, 529 strains) and $P$. aeruginosa (6.9\%, 297 strains) (Table 1). We also investigated the distribution of strains from HA $(n=2765,72.16 \%)$ and CA $(n=1039,27.11 \%)(P<0.0001)$ infections, but most of the isolates were sampled from HA infections (62.59-80.42\%) (Table 1). Enterobacteriaceae were present in the majority of isolates and accounted for $88.5 \%$, including mainly E.coli (63.2\%), followed by K. pneumonia (12.2\%), P. mirabilis (3.4\%) and Enterobacter cloacae (E. cloacae) (3.3\%), while others were present at a rate $<1.3 \%$. Non-Enterobacteriaceae accounted for only $11.5 \%$ of all isolates and included mainly $P$. aeruginosa (6.9\%) and Acinetobacter baumannii (A. baumannii) (3.3\%).

\section{In vitro susceptibility of Enterobacteriaceae and non- Enterobacteriaceae during 2010-2014}

Among the 12 analyzed antimicrobial agents, the susceptibility rates of ertapenem and imipenem against $E$. coli over 5 years were $96.4 \%(2,639 / 2,737)$ and $98.7 \%(2,702 /$ 2,737), with $\mathrm{MIC}_{90}$ values of $0.25 \mu \mathrm{g} / \mathrm{mL}$ for both drugs. Most E.coli isolates remained susceptible to amikacin (92.8\%) and piperacillin-tazobactam (92.51\%). However, the susceptibilities to third- and fourth-generation cephalosporins were relatively low, with rates of 58.5, 38.2, 34.6 and $34.4 \%$ for ceftazidime (CAZ), cefepime (FEP), cefotaxime (CTX) and ceftriaxone (CRO), respectively. The susceptibility rates of $E$. coli to fluoroquinolones and ampicillin-sulbactam were also less than 30 and $20 \%$, respectively (Fig. 1, Table 2).

Against $K$. pneumonia, the most potent antibiotics were imipenem (92.6\% susceptibility), amikacin (89.2\% susceptibility) and ertapenem (87.9\% susceptibility), with $\mathrm{MIC}_{90}$ values of $1 \mu \mathrm{g} / \mathrm{mL},>32 \mu \mathrm{g} / \mathrm{mL}$ and $1 \mu \mathrm{g} / \mathrm{mL}$, respectively. Piperacillin-tazobactam was the fourth most active agent, with a susceptibility of $75.8 \%$. The susceptibility rates of other antibiotics ranged from 30.6\% (ampicillin-sulbactam) to $67.5 \%$ (cefoxitin) (Fig. 1, Table 2,).

Against P. mirabilis, antimicrobial agents with $>90 \%$ susceptibility rates included ertapenem (99.3\%), piperacillin-tazobactam (99.3\%) and amikacin (91.2\%), but in HA infections, a $>90 \%$ susceptibility rate was found for ceftazidime (90.2\%). Cephalosporin susceptibility rates were $55.8-88.4 \%$ whereas fluoroquinolones exhibited $41.5-55.1 \%$ activity. Imipenem had poor

Table 1 Distribution of the UTI pathogens in China between 2010 and 2014

\begin{tabular}{|c|c|c|c|c|c|}
\hline & Total & CA (n/\% of total) & HA (n/\% of total) & Not identified (n/\% of total) & $P$-value \\
\hline Enterobacteriaceae & 3,832 & $1,039(27.11)$ & $2,765(72.16)$ & $28(0.73)$ & $<0.0001$ \\
\hline Escherichia coli & 2,737 & $739(27.00)$ & $1,976(72.20)$ & $22(0.80)$ & $<0.0001$ \\
\hline Klebsiella pneumoniae & 529 & $129(24.39)$ & $398(75.24)$ & $2(0.38)$ & $<0.0001$ \\
\hline Proteus mirabilis & 147 & 54 (36.73) & $92(62.59)$ & $1(0.68)$ & 0.011 \\
\hline Enterobacter cloacae & 141 & $39(27.66)$ & $101(71.63)$ & $1(0.71)$ & $<0.0001$ \\
\hline Citrobacter freundii & 54 & $11(20.37)$ & $43(79.63)$ & $0(0.00)$ & 0.0003 \\
\hline Klebsiella oxytoca & 51 & $18(35.29)$ & $33(64.71)$ & $0(0.00)$ & 0.1205 \\
\hline other & 173 & $49(28.32)$ & $122(70.52)$ & $2(1.16)$ & $<0.0001$ \\
\hline Non-Enterobacteriaceae & 500 & $105(21.00)$ & $391(78.2)$ & $4(0.8)$ & $<0.0001$ \\
\hline Pseudomonas aeruginosa & 297 & $65(21.89)$ & 231 (77.78) & $1(0.34)$ & $<0.0001$ \\
\hline Acinetobacter baumannii & 143 & $26(18.18)$ & $115(80.42)$ & $2(1.40)$ & $<0.0001$ \\
\hline other & 60 & $14(23.33)$ & $45(75.00)$ & $1(1.67)$ & $<0.0001$ \\
\hline All & 4,332 & $1,144(26.41)$ & $3,156(72.85)$ & $32(0.74)$ & $<0.0001$ \\
\hline
\end{tabular}

Not identified: A total of 32 isolates lacked partial demographic information and could not be identified as CA or HA isolates. They were not included in further analyses 


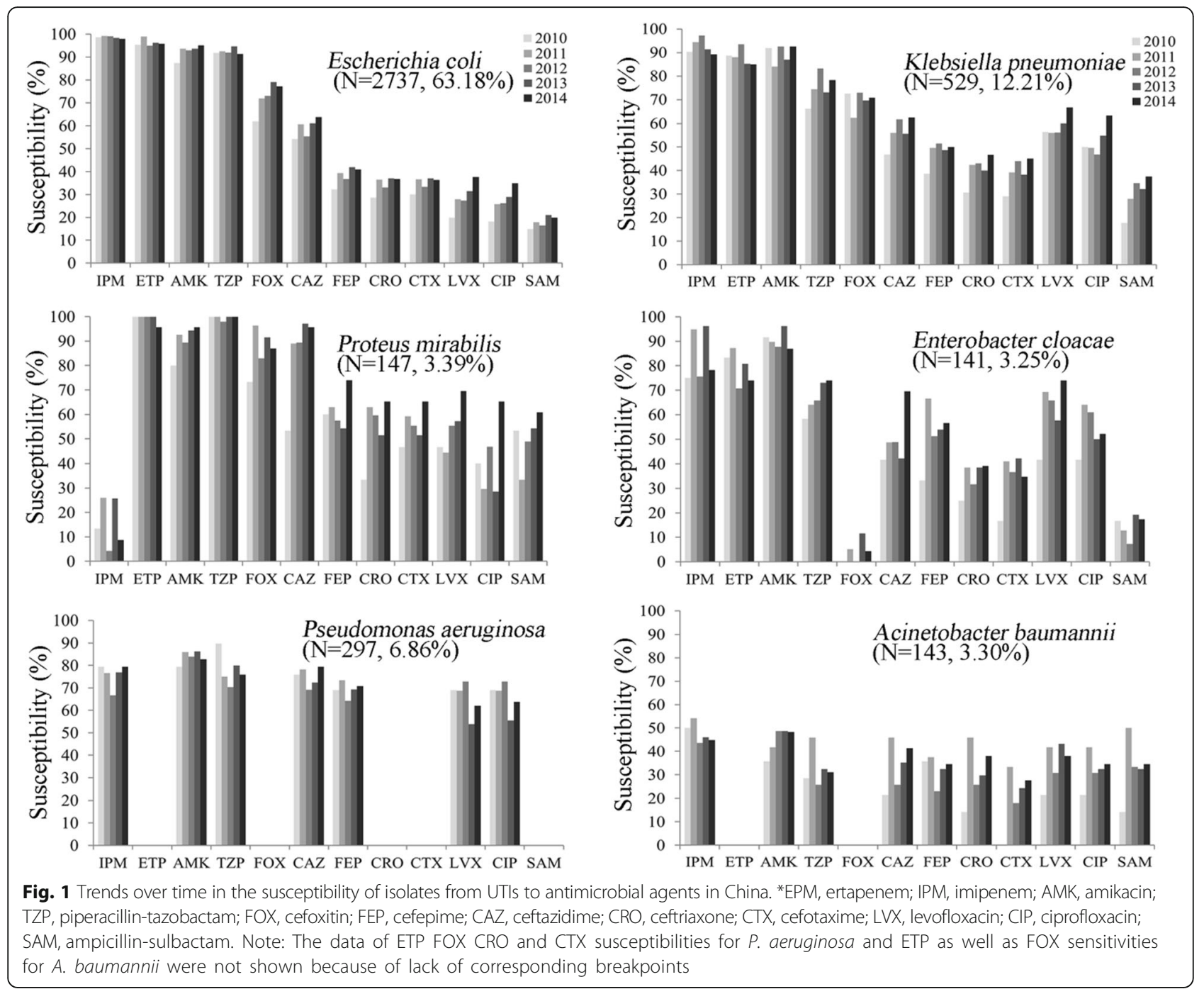

activity against $P$. mirabilis isolates, with a mean susceptibility rate of only $15.0 \%$ in both $\mathrm{CA}$ and $\mathrm{HA}$ derived isolates (Figs. 1 and 2, Table 2).

Antimicrobial resistance in Enterobacter cloacae was more pronounced than in E. coli and K. pneumonia. The antimicrobial agents with susceptibility rates of $>80 \%$ were amikacin $(90.1 \%)$ and imipenem $(85.1 \%)$ over the 5 -year study period. Particularly in 2014, ertapenem and piperacillin-tazobactam susceptibility rates in HA infections dropped to $53.9 \%$, whereas CA UTIs were still $100 \%$ susceptible to both antibiotics (Fig. 2). However, ertapenem was the third most active agent with susceptibilities of $78.7 \%$ in all isolates, followed by piperacillintazobactam (67.4\%), levofloxacin (64.5\%), ciprofloxacin (56.7\%) and cefepime (55.3\%) (Fig. 1, Table 2).

Although non-Enterobacteriaceae did not show high susceptibilities to the 12 common antibiotics, amikacin exhibited the highest in vitro activity against $P$. aeruginosa, with a susceptibility rate of $84.2 \%$ over the 5 -year study period, followed by piperacillin-tazobactam, imipenem, ceftazidime, cefepime, ciprofloxacin, and levofloxacin. (Figure 1, Table 2).

A. baumannii was the second most frequently isolated non-fermentative Gram-negative bacillus, comprising $3.3 \%(143 / 4,332)$ of all UTIs. The most active agents against $A$. baumannii were imipenem and amikacin, with susceptibility rates of 46.9 and $46.2 \%$, respectively over the entire study period. The other analyzed agents were less effective, with susceptibility rates of $<40 \%$ (Fig. 1, Table 2).

The trend of extended spectrum beta-lactamases (ESBL) producing bacteria occurrence in UTIs from 2010 to 2014

Figure 2a-c shows the frequency of ESBL-producing E.coli, K. pneumonia, K. oxytoca and P. mirabilis strains over the study period. The percentage of ESBL positive E. coli isolates decreased from $68.6 \%$ in 2010 to $59.1 \%$ in 2014, while the ESBL rate in $K$. pneumonia decreased from 59.7 to $49.2 \%$ and in $P$. mirabilis from 40.0 to $26.1 \%$ during the 5 -year study period. The susceptibility 
Table 2 Susceptibilities of UTI pathogens isolated between 2010 and 2014

\begin{tabular}{|c|c|c|c|c|}
\hline & Antibiotics & $\mathrm{S} \%$ & $\begin{array}{l}\text { MIC50 } \\
(\mu \mathrm{g} / \mathrm{ml})\end{array}$ & $\begin{array}{l}\text { MIC90 } \\
(\mu \mathrm{g} / \mathrm{ml})\end{array}$ \\
\hline \multirow[t]{4}{*}{ Escherichia coli } & IPM & 98.72 & 0.12 & 0.25 \\
\hline & ETP & 96.42 & $\leq 0.03$ & 0.25 \\
\hline & AMK & 92.88 & $\leq 4$ & 16 \\
\hline & TZP & 92.51 & $\leq 2$ & 16 \\
\hline \multirow[t]{4}{*}{ Klebsiella pneumoniae } & IPM & 92.63 & 0.25 & 1 \\
\hline & AMK & 89.22 & $\leq 4$ & $>32$ \\
\hline & ETP & 87.9 & $\leq 0.03$ & 1 \\
\hline & TZP & 75.8 & $\leq 2$ & $>64$ \\
\hline \multirow[t]{4}{*}{ Proteus mirabilis } & ETP & 99.32 & $\leq 0.03$ & 0.06 \\
\hline & TZP & 99.32 & $\leq 2$ & 4 \\
\hline & AMK & 91.16 & 8 & 16 \\
\hline & $C A Z$ & 88.44 & $\leq 0.5$ & 8 \\
\hline \multirow[t]{4}{*}{ Enterobacter cloacae } & AMK & 90.07 & $\leq 4$ & 16 \\
\hline & IPM & 85.11 & 0.5 & 2 \\
\hline & ETP & 78.72 & 0.12 & 4 \\
\hline & TZP & 67.38 & 4 & $>64$ \\
\hline \multirow[t]{4}{*}{ Pseudomonas aeruginosa } & AMK & 84.18 & 8 & $>32$ \\
\hline & TZP & 76.43 & 4 & $>64$ \\
\hline & IPM & 74.75 & 1 & $>8$ \\
\hline & CAZ & 74.41 & 4 & 64 \\
\hline \multirow[t]{4}{*}{ Acinetobacter baumannii } & IPM & 46.85 & 8 & $>8$ \\
\hline & AMK & 46.15 & $>32$ & $>32$ \\
\hline & LVX & 36.36 & $>4$ & $>4$ \\
\hline & CAZ & 34.27 & 64 & $>128$ \\
\hline
\end{tabular}

differences to ertapenem and imipenem between ESBL and non-ESBL producing strains were generally small, but were greater for other agents, particularly for the thirdand fourth-generation cephalosporins, including ceftriaxone $(1.1 \%$ against ESBL-producing isolates vs $91.0 \%$ against ESBL-non-producing isolates), ceftazidime $(38.4 \%$ vs $93.5 \%$ ) and cefepime (4.5\% vs $96.7 \%$ ) (data not shown).

Figure 2d-e shows the ESBL rates in E. coli, K. pneumonia, and P. mirabilis from UTIs in different regions in China. We categorized the 21 participating sites into 5 different regions in China (Northeast (Haerbin, Changchun and Shenyang), North (Beijing and Tianjing), East (Hangzhou, Nanjing, Jinan, Nanchang and Shanghai), South (Chongqing, Guangzhou and Haikou) and Central China (Changsha, Zhengzhou and Wuhan)). The two sites in the Central China region exhibited higher ESBL rates in E. coli (81.5\%) and K. pneumonia (64.9\%), while other regions showed relatively lower ESBL rates in these two species $(54.5-65.1 \%$ for E. coli, and $48.1-56.3 \%$ for K. pneumoniae). For P. mirabilis, the
ESBL rates ranged from $31.4 \%$ (South China region) to 47.5\% (North China region).

\section{Discussion}

Nitrofurantoin, trimethoprim-sulfamethoxazole, fosfomycin, fluoroquinolones and beta-lactams are commonly recommended antimicrobial agents for urinary tract infections [14]. However, fosfomycin and nitrofurantoin are not often used in China [2]. The usage of trimethoprim-sulfamethoxazole for the treatment of UTIs in China is also limited because of a high resistance rate to this agent among E.coli isolates [15]. In view of this finding, we focused on the activity of betalactams, fluoroquinolones and aminoglycoside against uropathogens in the present study. Since Enterobacteriaceae accounted for the majority of aerobic and facultative anaerobic pathogens causing UTIs $(88.5 \%$ of all isolates) in our study, with E.coli, K. pneumonia, P. mirabilis and Enterobacter cloacae the most frequently isolated species, knowledge of their resistance pattern is beneficial.

Cephalosporins are commonly recommended as empirical choices for UTIs, but their efficacy is greatly reduced when the pathogens produce ESBL. Over the entire study period, susceptibility rates of Enterobacteriaceae to third-generation and fourth-generation cephalosporins were $51.4-66.0 \%$ for ceftazidime, 29.4-46.9\% for cefotaxime, $29.9-41.2 \%$ for ceftriaxone and $35.1-$ $47.1 \%$ for cefepime, indicating that these agents might not be the optimum medications for empirical UTI therapies. In the present study, the percentage of ESBL positive E. coli isolates decreased from $66.9 \%$ in 2010 to $59.1 \%$ in 2014, while for K. pneumonia it decreased from 59.7 to $48.8 \%$ and from 40.0 to $26.1 \%$ among $P$. mirabilis. The data were well matched with the nonsusceptibility rates to cephalosporins against each species, which indicated that ESBL production might be a reason for cephalosporin resistance [16]. The decrease of ESBL rates in E. coli, K. pneumonia and P. mirabilis may have been a result of China's antimicrobial stewardship policy on antimicrobial use, which has been promoted for a number of years [17-19]. Our study also highlighted the variation in ESBL rates in different regions of China, with the Central-China region having a higher ESBL prevalence in E. coli and K. pneumonia. Researchers previously reported that the ESBL genotypes in China were mainly CTX-M types [20-22], especially CTX-M-14, -15, and -55 for E. coli and K. pneumonia, and CTX-M-65 and -14 for P. mirabilis [22]. Plasmids encoding these CTX-M enzymes reached human opportunists, where they have proliferated in community $E$. coli and hospital $K$. species. CTX-M families are dominate in different regions: CTX-M-15 is predominant in most of Europe, North America, the Middle East, and India, but 


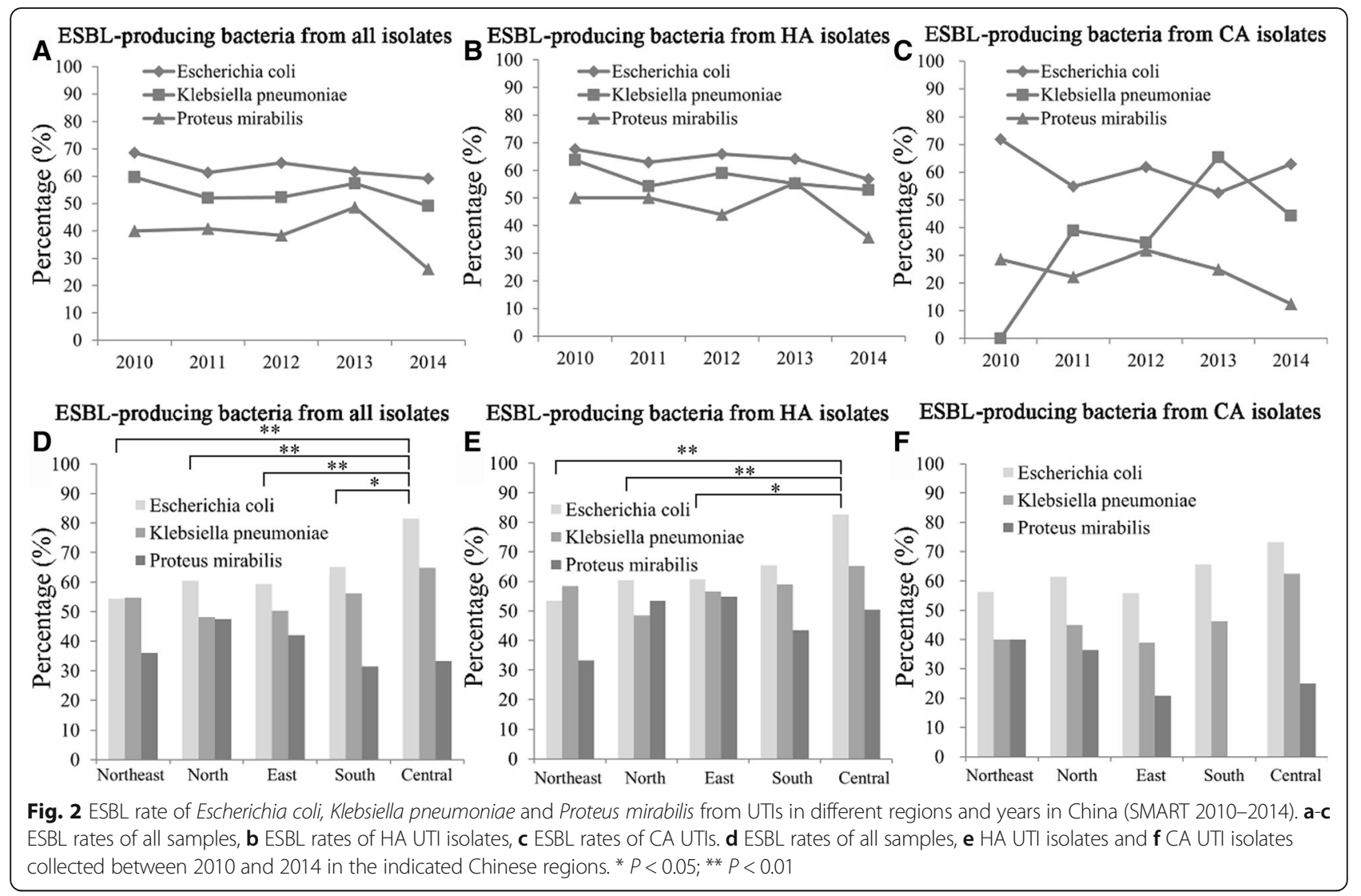

CTX-M-14 is most common in China, Southeast Asia and Spain, while CTX-M-2 is predominant in Argentina, Israel, and Japan [23, 24]. Increased numbers of enzyme types and prevalence made determination of resistance profiles more complicated.

Fluoroquinolones, especially ciprofloxacin and levofloxacin, were considered to be effective antimicrobial agents against uropathogens because of high drug concentrations are reached in the urine. However, fluoroquinolone-resistant $E$. coli is also problematic in China. The susceptibility of $E$. coli to fluoroquinolones (ciprofloxacin and levofloxacin) was 26.9-28.9\%, with rates of $30.2-32.1 \%$ against $\mathrm{CA}$ isolates and 25.7-27.8\% against $\mathrm{HA}$ isolates. Wang et al. also previously reported about ciprofloxacin-resistant E. coli strains with multiple gyrA and parC gene substitutions [25]. Regarding the low effectiveness of fluoroquinolones against Enterobacteriaceae, ciprofloxacin and levofloxacin should not be considered as first line agents for empirical therapies of complicated UTIs. Our data also showed that susceptibilities of ESBLproducing E. coli and K. pneumonia strains to fluoroquinolones were significantly lower than that of ESBL-non-producing strains, which is in agreement with previous findings [26].
Carbapenems can still be considered to be suitable for severe infections and as alternative empiric treatment for UTIs caused by bacterial strains highly suspicious of being ESBL-producing or AmpC-derepressed Enterobacteriaceae [27-29]. Although carbapenems were not the first line choices for uncomplicated cystitis and pyelonephritis in women according to the IDSA guideline, they were good alternatives against multidrug resistant Gram-negative bacilli that caused UTIs. Our study showed that ertapenem and imipenem were the most effective agents against Enterobacteriaceae causing UTIs, with susceptibility rates of $92.5-96.5 \%$ and $89.9-95.2 \%$, respectively (2010-2014). On the other hand, carbapenem-resistant Enterobacteriaceae have emerged, which has also been noted in other reports [30-33], especially KPC-producing $K$. pneumonia in the northeastern area of the United States of America [31], KPC/ VIM-producing Enterobacteriaceae in Greece [32, 33] and $\mathrm{KPC}$-producing isolates in eastern China. In our study, very few E. coli isolates $(<4 \%)$ were nonsusceptible to carbapenems, while there was a certain proportion of carbapenem-non-susceptible $K$. pneumonia isolates (13.8\% to ertapenem), P. mirabilis (85\% to imipenem) and E. cloacae (21.3\% to ertapenem and $14.9 \%$ to imipenem), which should be noted by 

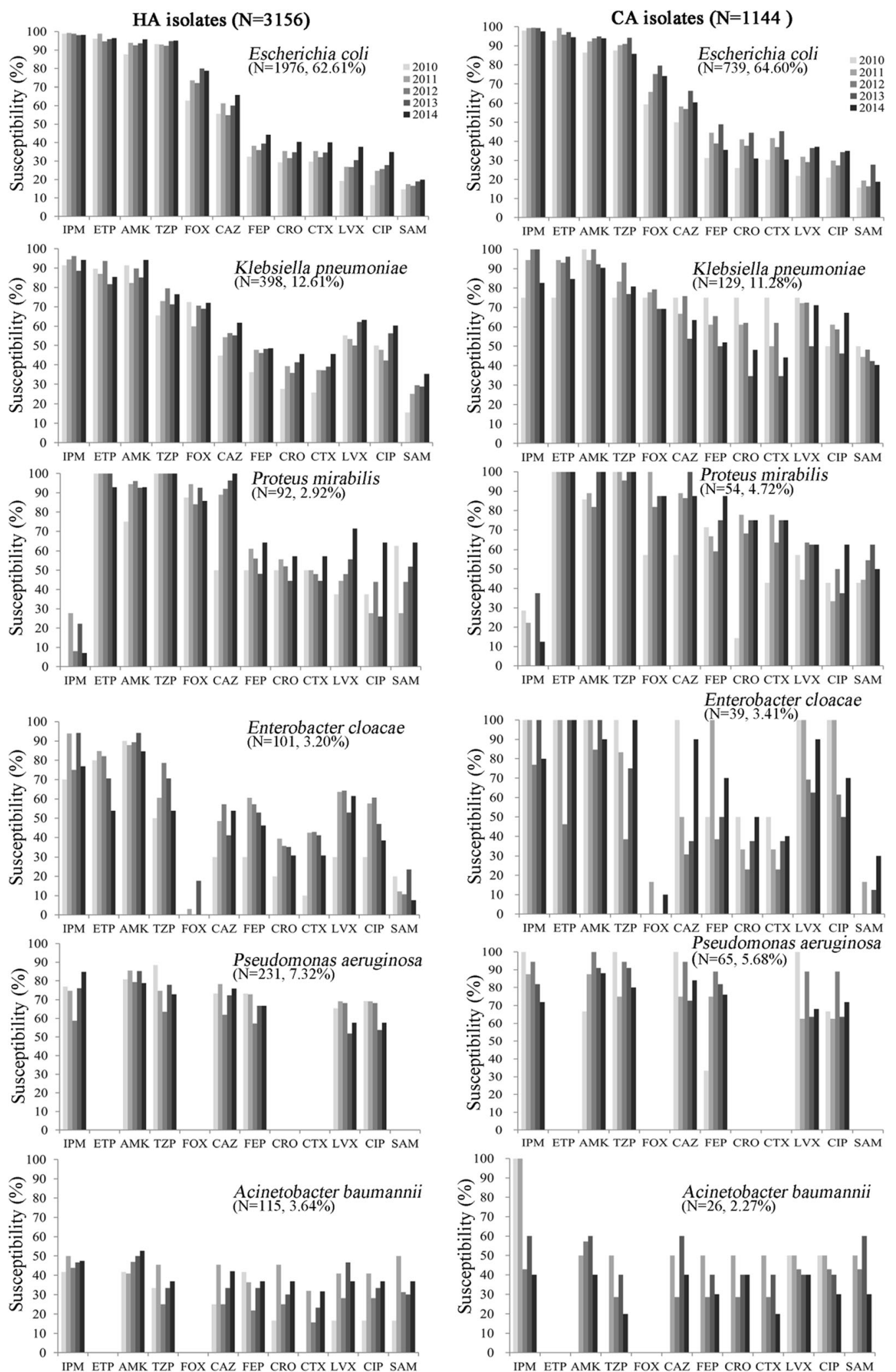

Fig. 3 (See legend on next page.) 
(See figure on previous page.)

Fig. 3 Trends over time in the susceptibility of isolates from UTIs to antimicrobial agents in China (CA and HA). *EPM, ertapenem; IPM, imipenem; AMK, amikacin; TZP, piperacillin-tazobactam; FOX, cefoxitin; FEP, cefepime; CAZ, ceftazidime; CRO, ceftriaxone; CTX, cefotaxime; LVX, levofloxacin; CIP, ciprofloxacin; SAM, ampicillin-sulbactam. Note: The data of ETP FOX CRO and CTX susceptibilities for P. aeruginosa and ETP as well as FOX sensitivities for A. baumannii were not shown because of lack of corresponding breakpoints

clinicians. Especially for E. cloacae the susceptibility of HA samples to ertapenem has dropped to $53.9 \%$, while for CA UTIs its susceptibility rate is $100 \%$. Hospital infections caused by E. cloacae, which is a typical commensal under normal conditions, have been suggested to be mainly caused by endogenous translocation from the digestive tract in debilitated patients and that under antibiotic therapy, E. cloacae strains may selectively reproduce excessively in the gastrointestinal tract [34]. This might be the reason for the high ertapenem resistance in UTIs mainly caused by HA E. cloacae. The main resistance mechanism to carbapenem in Enterobacteriaceae was reported to be carbapenemase production and porin loss in China [35]. However, the resistance of $P$. mirabilis to imipenem was caused by a mechanism other than carbapenemase [13].

Among the tested antimicrobial agents, amikacin exhibited good activity against most of the uropathogens (80.096.2\% susceptibility rate against Enterobacteriaceae and 83.6\% against $P$. aeruginosa). Although the use of this aminoglycoside is limited because of its toxicity, it has also been recommended as an alternative to carbapenems against ESBL-producing isolates that cause UTIs [36].

\section{Conclusion}

Carbapenems remain the most effective antimicrobial agents against UTI Gram-negative pathogens, followed by amikacin and piperacillin-tazobactam in China between 2010 and 2014. Due to the reduced susceptibility of Enterobacteriaceae to cephalosporins and fluoroquinolones, we recommend that these antibiotics should not be used for empirical UTI therapies in China.

\section{Abbreviations \\ AMK: Amikacin; CA: Community acquired; CAZ: Ceftazidime; CIP: Ciprofloxacin; CLSI: Clinical and Laboratory Standards Institute; CRO: Ceftriaxone; CTX: Cefotaxime; EPM: Ertapenem; ESBLs: Extended spectrum beta-lactamases; FEP: Cefepime; FOX: Cefoxitin; HA: Hospital acquired; IPM: Imipenem; LVX: Levofloxacin; MICs: Minimum inhibitory concentrations; SAM: Ampicillin-sulbactam; SMART: Study for monitoring antimicrobial resistance trends; TZP: Piperacillin-tazobactam; UTIs: Urinary tract infections}

\section{Acknowledgements}

We thank all investigators involved in this study. We also thank Shanghai BIOMED Science Technology (Shanghai, China) which was funded by MSD China for providing medical editorial assistance.

\section{Funding}

This study was funded by Merck Sharp \& Dohme (MSD; Whitehouse Station, NJ, USA) and this study was also funded by CAMS Initiative for Innovative Medicine (2016-12M-3-014).

\section{Availability of data and materials}

The data that support the findings of this study are available from MSD China Holding Co. Ltd. Data are however available from the authors upon reasonable request and with permission of MSD China Holding Co. Ltd.

\section{Authors' contributions}

QWY, HZ and YCX were responsible for the conception and design of the study, drafted the manuscript and revised and commented the draft; QWY, $H Z, Y W, Z P X, G Z, X X C, Y C X, B C, H S K, Y X N, Y S Y, Z Y S, B J H, W X H, Y W, A H W, X J F$, $\mathrm{KL}, \mathrm{YPL}, \mathrm{ZDH}, \mathrm{YZC}$, JL, JRS, BDG, QD, SFZ, HFS and REB performed the data analysis. All authors read and approved the final version of the manuscript and confirm that the content has not been published elsewhere and does not overlap with or duplicate their published work.

\section{Competing interests}

The authors declare that they have no competing interests.

\section{Consent for publication}

Not applicable.

\section{Ethics approval and consent to participate}

All participating institutions agreed to comply with Investigational Review Boards, Good Clinical Practices, and Good Laboratory Practices. The approval of each hospital Investigational Review Board (IRB) has been obtained prior to the start of the investigation, depending on the IRB policies at the institutions.

\section{Author details}

'Department of Clinical Laboratory, Peking Union Medical College Hospital, Peking Union Medical College, Chinese Academy of Medical Sciences, Beijing 100730, China. ${ }^{2}$ Department of Respiratory and Critical Care Medicine, Clinical Microbiology and Infectious Disease Lab., China-Japan Friendship Hospital, Beijing 100029, China. ${ }^{3}$ Department of Microbiology, The First Affiliated Hospital of Zhejiang University, Hangzhou 310003, China. ${ }^{4}$ Division of Microbiology, Ruijin Hospital, School of Medicine, Shanghai Jiaotong University, Shanghai 200025, China. ${ }^{5}$ Department of Infectious Diseases, SirRunRun Shaw Hospital, School of Medicine, Zhejiang University, Hangzhou 310016, China. ' Department of Laboratory Medicine, Tongji Hospital, Tongji Medical College, Huazhong University of Science and Technology, Wuhan 430030, China. ${ }^{7}$ Division of Microbiology, Zhongshan Hospital of Fudan University, Shanghai 200032, China. ${ }^{8}$ Division of Microbiology, The First Affiliated Hospital of Chongqing Medical University, Chongqing 400016, China. ${ }^{9}$ Department of Laboratory Medicine, Shandong Provincial Hospital Affiliated to Shandong University, Jinan 250021, China. ${ }^{10}$ Infection control center, Xiangya Hospital, Central South University, Changsha 410008, China. ${ }^{11}$ Division of Microbiology, The First Affiliated Hospital of Zhengzhou University, Zhenzhou 450052, China. ${ }^{12}$ Division of Microbiology, The First Affiliated Hospital, Sun Yat-Sen University, Guangzhou 510080, China. ${ }^{13}$ Department of Microbiology, The Chinese PLA General Hospital, Beijing 100853, China. ${ }^{14}$ Division of Microbiology, Tianjin Medical University General Hospital, Tianjing 300052, China. ${ }^{15}$ Division of Microbiology, The First Affiliated Hospital of Chinese Medical University, Shenyang 110001, China. ${ }^{16}$ Department of Clinical Laboratory, The First Affiliated Hospital of Harbin Medical University, Harbin 150001, China. ${ }^{17}$ Department of Clinical Laboratory, Beijing Friendship Hospital of Capital Medical University, Beijing 100020, China. ${ }^{18}$ Clinical Laboratory, The Second Affiliated Hospital of Nanchang University, Nanchang 330006, China. ${ }^{19}$ Microbiology Lab, Jilin Province People's Hospital, Changchun 130021, China. ${ }^{20}$ Division of Microbiology, Haikou People's Hospital, Haikou 570208, China. ${ }^{21}$ Division of Microbiology, General Hospital of Nanjing Military Command, Nanjing 210002, China. ${ }^{22}$ Division of Microbiology, International Health Management Associates, Schaumburg, IL 60173-3817, USA. 
Received: 29 November 2016 Accepted: 28 February 2017 Published online: 06 March 2017

\section{References}

1. Bi XC, Zhang B, Ye YK, He HC, Han ZD, Dai QS, et al. Pathogen incidence and antibiotic resistance patterns of catheter-associated urinary tract infection in children. J Chemother. 2009;21:661-5.

2. Qiao LD, Chen S, Yang Y, Zhang K, Zheng B, Guo HF, et al. Characteristics of urinary tract infection pathogens and their in vitro susceptibility to antimicrobial agents in China: data from a multicenter study. BMJ Open. 2013;3:e004152.

3. Hertz FB, Schonning K, Rasmussen SC, Littauer P, Knudsen JD, LobnerOlesen A, et al. Epidemiological factors associated with ESBL- and non ESBLproducing E. coli causing urinary tract infection in general practice. Infect Dis (Lond). 2016:48:241-5.

4. Guembe M, Cercenado E, Alcala L, Marin M, Insa R, Bouza E. Evolution of antimicrobial susceptibility patterns of aerobic and facultative gramnegative bacilli causing intra-abdominal infections: results from the SMART studies 2003-2007. Rev Esp Quimioter. 2008;21:166-73.

5. Stapleton AE. Urinary tract infection pathogenesis: host factors. Infect Dis Clin North Am. 2014;28:149-59.

6. Foxman B. Urinary tract infection syndromes: occurrence, recurrence, bacteriology, risk factors, and disease burden. Infect Dis Clin North Am. 2014;28:1-13.

7. Wellington EM, Boxall AB, Cross P, Feil EJ, Gaze WH, Hawkey PM, et al. The role of the natural environment in the emergence of antibiotic resistance in gram-negative bacteria. Lancet Infect Dis. 2013;13:155-65.

8. Caccamo M, Perilli M, Celenza G, Bonfiglio G, Tempera G, Amicosante G. Occurrence of extended spectrum beta-lactamases among isolates of Enterobacteriaceae from urinary tract infections in southern Italy. Microb Drug Resist. 2006;12:257-64.

9. Al Yousef SA, Younis S, Farrag E, Moussa H, Bayoumi FS, Ali AM. Clinical and laboratory profile of urinary tract infections associated with extended Spectrum beta-Lactamase producing Escherichia coli and Klebsiella pneumoniae. Ann Clin Lab Sci. 2016:46:393-400.

10. Bonkat G, Muller G, Braissant O, Frei R, Tschudin-Suter S, Rieken M, et al. Increasing prevalence of ciprofloxacin resistance in extended-spectrumbeta-lactamase-producing Escherichia coli urinary isolates. World J Urol. 2013;31:1427-32

11. Hawser SP, Bouchillon SK, Hoban DJ, Badal RE. In vitro susceptibilities of aerobic and facultative anaerobic Gram-negative bacilli from patients with intra-abdominal infections worldwide from 2005-2007: results from the SMART study. Int J Antimicrob Agents. 2009;34:585-8.

12. Institute CaLS. Method for dilution antimicrobial susceptibility tests for bacteria that grow aerobically; approved standard. 9th ed. Wayne: PA:CLSI document M7-A9; 2012.

13. Institute CaLS. Performance standards for antimicrobial susceptibility testing. Twenty-fifth informational supplement. Wayne: PA:Document M100-S25: 2015.

14. Gupta K, Hooton TM, Naber KG, Wullt B, Colgan R, Miller LG, et al. International clinical practice guidelines for the treatment of acute uncomplicated cystitis and pyelonephritis in women: a 2010 update by the infectious diseases Society of America and the European society for microbiology and infectious diseases. Clin Infect Dis. 2011;52:e103-20.

15. Zhao L, Chen X, Zhu X, Yang W, Dong L, Xu X, et al. Prevalence of virulence factors and antimicrobial resistance of uropathogenic Escherichia coli in Jiangsu province (China). Urology. 2009;74:702-7.

16. Hope R, Potz NA, Warner M, Fagan EJ, Arnold E, Livermore DM. Efficacy of practised screening methods for detection of cephalosporin-resistant Enterobacteriaceae. J Antimicrob Chemother. 2007;59:110-3.

17. Guo W, He Q, Wang Z, Wei M, Yang Z, Du Y, et al. Influence of antimicrobial consumption on gram-negative bacteria in inpatients receiving antimicrobial resistance therapy from 2008-2013 at a tertiary hospital in Shanghai, China. Am J Infect Control. 2015;43:358-64.

18. Ding H, Yang Y, Wei J, Fan S, Yu S, Yao K, et al. Influencing the use of antibiotics in a Chinese pediatric intensive care unit. Pharm World Sci. 2008; 30:787-93.

19. Zou YM, Ma Y, Liu JH, Shi J, Fan T, Shan YY, et al. Trends and correlation of antibacterial usage and bacterial resistance: time series analysis for antibacterial stewardship in a Chinese teaching hospital (2009-2013). Eur J Clin Microbiol Infect Dis. 2015;34:795-803.
20. Chanawong A, M'Zali FH, Heritage J, Xiong JH, Hawkey PM. Three cefotaximases, CTX-M-9, CTX-M-13, and CTX-M-14, among Enterobacteriaceae in the People's Republic of China. Antimicrob Agents Chemother. 2002:46:630-7.

21. Wang H, Kelkar S, Wu W, Chen M, Quinn JP. Clinical isolates of Enterobacteriaceae producing extended-spectrum beta-lactamases: prevalence of CTX-M-3 at a hospital in China. Antimicrob Agents Chemother. 2003;47:790-3.

22. Yang $\mathrm{Q}$, Zhang $\mathrm{H}$, Cheng J, Xu Z, Xu Y, Cao B, et al. In vitro activity of flomoxef and comparators against Escherichia coli, Klebsiella pneumoniae and Proteus mirabilis producing extended-spectrum beta-lactamases in China. Int J Antimicrob Agents. 2015;45:485-90.

23. Hawkey PM. Prevalence and clonality of extended-spectrum betalactamases in Asia. Clin Microbiol Infect. 2008;14 Suppl 1:159-65.

24. Bush K. Extended-spectrum beta-lactamases in North America, 1987-2006. Clin Microbiol Infect. 2008;14 Suppl 1:134-43.

25. Wang H, Dzink-Fox JL, Chen M, Levy SB. Genetic characterization of highly fluoroquinolone-resistant clinical Escherichia coli strains from China: role of acrR mutations. Antimicrob Agents Chemother. 2001;45:1515-21.

26. Ben-Ami R, Rodriguez-Bano J, Arslan H, Pitout JD, Quentin C, Calbo ES, et al A multinational survey of risk factors for infection with extended-spectrum beta-lactamase-producing enterobacteriaceae in nonhospitalized patients. Clin Infect Dis. 2009;49:682-90.

27. Livermore DM, Oakton KJ, Carter MW, Warner M. Activity of ertapenem (MK0826) versus Enterobacteriaceae with potent beta-lactamases. Antimicrob Agents Chemother. 2001;45:2831-7.

28. Essack SY. Treatment options for extended-spectrum beta-lactamaseproducers. FEMS Microbiol Lett. 2000;190:181-4.

29. Paterson DL. Recommendation for treatment of severe infections caused by Enterobacteriaceae producing extended-spectrum beta-lactamases (ESBLs). Clin Microbiol Infect. 2000;6:460-3.

30. Kaczmarek FM, Dib-Hajj F, Shang W, Gootz TD. High-level carbapenem resistance in a Klebsiella pneumoniae clinical isolate is due to the combination of bla(ACT-1) beta-lactamase production, porin OmpK35/36 insertional inactivation, and down-regulation of the phosphate transport porin phoe. Antimicrob Agents Chemother. 2006;50:3396-406.

31. Bratu S, Landman D, Haag R, Recco R, Eramo A, Alam M, et al. Rapid spread of carbapenem-resistant Klebsiella pneumoniae in New York City: a new threat to our antibiotic armamentarium. Arch Intern Med. 2005;165:1430-5.

32. Vatopoulos A. High rates of metallo-beta-lactamase-producing Klebsiella pneumoniae in Greece-a review of the current evidence. Euro Surveill. 2008;13.

33. Maltezou HC, Giakkoupi P, Maragos A, Bolikas M, Raftopoulos V, Papahatzaki

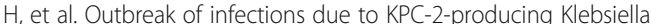
pneumoniae in a hospital in Crete (Greece). J Infect. 2009;58:213-9.

34. Keller R, Pedroso MZ, Ritchmann R, Silva RM. Occurrence of virulenceassociated properties in Enterobacter cloacae. Infect Immun. 1998;66:645-9.

35. Yang $Q$, Wang $H$, Sun $H$, Chen $H, X u Y$, Chen M. Phenotypic and genotypic characterization of Enterobacteriaceae with decreased susceptibility to carbapenems: results from large hospital-based surveillance studies in China. Antimicrob Agents Chemother. 2010;54:573-7.

36. Han SB, Lee SC, Lee SY, Jeong DC, Kang JH. Aminoglycoside therapy for childhood urinary tract infection due to extended-spectrum beta-lactamaseproducing Escherichia coli or Klebsiella pneumoniae. BMC Infect Dis. 2015; $15: 414$

\section{Submit your next manuscript to BioMed Central} and we will help you at every step:

- We accept pre-submission inquiries

- Our selector tool helps you to find the most relevant journal

- We provide round the clock customer support

- Convenient online submission

- Thorough peer review

- Inclusion in PubMed and all major indexing services

- Maximum visibility for your research

Submit your manuscript at www.biomedcentral.com/submit 